\title{
CCNE1 amplification and centrosome number abnormality in serous tubal intraepithelial carcinoma: further evidence supporting its role as a precursor of ovarian high-grade serous carcinoma
}

\author{
Elisabetta Kuhn ${ }^{1}$, Tian-Li Wang ${ }^{1}$, Kai Doberstein ${ }^{2}$, Asli Bahadirli-Talbott ${ }^{1}$, Ayse Ayhan ${ }^{1,3}$, \\ Ann Smith Sehdev ${ }^{4}$, Ronny Drapkin ${ }^{2}$, Robert J Kurman ${ }^{1,5}$ and Ie-Ming Shih ${ }^{1,5}$ \\ ${ }^{1}$ Department of Pathology, Johns Hopkins Medical Institutions, Baltimore, MD, USA; ${ }^{2}$ Department of \\ Obstetrics and Gynecology, Penn Ovarian Cancer Research Center, University of Pennsylvania, Philadelphia, \\ PA, USA; ${ }^{3}$ Department of Pathology, Seirei Mikatahara Hospital, Hamamatsu, Japan; ${ }^{4}$ Legacy Health Systems, \\ Portland, OR, USA and ${ }^{5}$ Department of Gynecology and Obstetrics, Johns Hopkins Medical Institutions, \\ Baltimore, MD, USA
}

\begin{abstract}
Aberration in chromosomal structure characterizes almost all cancers and has profound biological significance in tumor development. It can be facilitated by various mechanisms including overexpression of cyclin E1 and centrosome amplification. As ovarian high-grade serous carcinoma has pronounced chromosomal instability, in this study we sought to determine whether increased copy number of CCNE1 which encodes cyclin E1 and centrosome amplification ( $>2$ copies) occurs in its putative precursor, serous tubal intraepithelial carcinoma. We found CCNE1 copy number gain/amplification in $8(22 \%)$ of 37 serous tubal intraepithelial carcinomas and 12 (28\%) of 43 high-grade serous carcinomas. There was a correlation in CCNE1 copy number between serous tubal intraepithelial carcinoma and high-grade serous carcinoma in the same patients $(P<0.001)$. There was no significant difference in the percentage of CCNE1 gain/amplification between serous tubal intraepithelial carcinoma and high-grade serous carcinoma $(P=0.61)$. Centrosome amplification was recorded in only $5(14 \%)$ of 37 serous tubal intraepithelial carcinomas, and in $10(40 \%)$ of 25 high-grade serous carcinomas. The percentage of cells with centrosome amplification was higher in high-grade serous carcinoma than in serous tubal intraepithelial carcinoma $(P<0.001)$. Induced expression of cyclin E1 increased the percentage of fallopian tube epithelial cells showing centrosome amplification. Our findings suggest that gain/amplification of CCNE1 copy number occurs early in tumor progression and precedes centrosome amplification. The more prevalent centrosome amplification in high-grade serous carcinoma than in serous tubal intraepithelial carcinoma supports the view that serous tubal intraepithelial carcinoma precedes the development of many high-grade serous carcinomas.
\end{abstract}

Modern Pathology (2016) 29, 1254-1261; doi:10.1038/modpathol.2016.101; published online 22 July 2016

Ovarian high-grade serous carcinoma is the leading cause of gynecologic cancer-related death in the United States. ${ }^{1}$ Over the last decade, one of the major

Correspondence: Dr E Kuhn, MD, Department of Pathology, Johns Hopkins Medical Institutions, Baltimore, MD, USA and Professor I-M Shih, MD, PhD, Department of Gynecology and Obstetrics, Johns Hopkins Medical Institutions, Johns Hopkins University, 1550 Orleans Street, CRB-2, Room 305, Baltimore, MD 201231, USA.

E-mails: elisabettakuhn@hotmail.it and ishih@jhmi.edu

Received 2 March 2016; revised 19 April 2016; accepted 22 April 2016; published online 22 July 2016 advances toward understanding the development of ovarian high-grade serous carcinoma was the recognition that a lesion in the fallopian tube, designated 'serous tubal intraepithelial carcinoma', is the most likely precursor. ${ }^{2-4}$ Serous tubal intraepithelial carcinomas are identified on hematoxylin-and-eosin stained sections based on morphology alone or in combination with immunostaining patterns of p53, Ki-67, laminin C1, and stathmin 1 immunoreactivity. ${ }^{5-8}$ Using a comprehensive sampling technique of the fallopian tubes (ie, the SEE-FIM protocol), ${ }^{9,10}$ investigators have identified serous 
tubal intraepithelial carcinomas in $50-60 \%$ of patients with concurrently high and in approximately up to $5 \%$ of women without ovarian cancer but who have a genetic predisposition to ovarian high-grade serous carcinoma at the time of risk reducing salpingo-oophorectomy. ${ }^{9,10}$ Besides those discovered at the time of risk reducing salpingooophorectomy, serous tubal intraepithelial carcinomas have also been reported as incidental findings in women undergoing hysterectomy and bilateral salpingo-oophorectomy for a variety of benign and malignant conditions. ${ }^{11-15}$ The tubal origin of highgrade serous carcinoma is supported by molecular and epidemiologic studies $6,9,16,17$ and genetically engineered mouse models. ${ }^{4,18,19}$ Despite the recent advances in elucidating the roles of serous tubal intraepithelial carcinoma in the early pathogenesis of high-grade serous carcinoma, the molecular events involved in the development of serous tubal intraepithelial carcinoma and progression from serous tubal intraepithelial carcinoma to high-grade serous carcinoma are still not completely understood. A lingering question is whether serous tubal intraepithelial carcinoma is a precursor of high-grade serous carcinoma or a metastasis. ${ }^{20}$

To further characterize serous tubal intraepithelial carcinoma, particularly in regard to the molecular events involved in the transition from serous tubal intraepithelial carcinoma to high-grade serous carcinoma, we studied two related markers, CCNE1 copy number and centrosome amplification, in a series of serous tubal intraepithelial carcinomas and highgrade serous carcinomas, many of which were concurrent in the same patient. We focused on CCNE1 because it encodes cyclin E1 which binds to cyclin-dependent kinase 2 (CDK2) and has an important role not only in cell-cycle progression (G1- to S-phase transition) but also in centrosome duplication, a tightly regulated process that maintains genetic stability. ${ }^{21}$ CCNE1 gene amplification is one of the most common molecular genetic alterations that characterize high-grade serous carcinoma, especially in those tumors that develop resistance to platinum-based chemotherapy, ${ }^{22-25}$ and overexpression of cyclin E1 can be detected in many high-grade serous carcinomas as well as in some serous tubal intraepithelial carcinomas. ${ }^{26}$ It has been recently reported that cyclin E1 upregulation occurs early in fallopian tube secretory cell transformation to highgrade serous carcinomas. ${ }^{27}$ As in many other types of solid tumors, amplification and overexpression of CCNE1 in high-grade serous carcinomas are associated with increased chromosomal instability and poor clinical outcome. ${ }^{28-30}$ One of the main mechanisms that contribute to the aggressive behavior of cancers with abundant cyclin E1 proteins is the promotion of aberrant centrosome duplication, where more than two centrosomes appear in a cell, creating chromosomal instability after cytokinesis. ${ }^{21,31}$ Chromosomal instability, in turn, fuels tumor evolution, as it provides an expanding repertoire of tumor subpopulations to develop drug resistance and other highly malignant phenotypes. Indeed, multivariate survival analysis of The Cancer Genome Atlas of ovarian cancer shows that a higher degree of chromosomal aberrations, reflecting a history of chromosomal instability, was significantly associated with a poor overall survival in high-grade serous carcinoma patients. ${ }^{32}$

In the current study, we used fluorescence in situ hybridization and immunofluorescence to analyze CCNE1 DNA copy number and centrosome number, respectively, on tissue sections and cells in culture. By comparing serous tubal intraepithelial carcinomas and high-grade serous carcinomas, we determined whether chromosomal instability, as reflected by both markers, occurred early in tumor progression, and also what was the temporal sequence for tumor cells to acquire these aberrations.

\section{Materials and methods}

\section{Case Selection}

A total of 43 high-grade serous carcinomas and 37 serous tubal intraepithelial carcinomas were retrieved from the pathology files of the Johns Hopkins Hospital (Baltimore, MD) and the Legacy Health System (Portland, OR). In this series, 19 cases contained both serous tubal intraepithelial carcinomas and high-grade serous carcinomas from the same patients. Among them, 11 patients had two discrete serous tubal intraepithelial carcinomas; resulting in a total of 30 serous tubal intraepithelial carcinomas that were concurrently associated with high-grade serous carcinomas and 7 serous tubal intraepithelial carcinomas that were not associated with high-grade serous carcinomas. Germline $B R C A 1 / 2$ status was not known. All the available slides were reviewed to confirm the diagnosis using the criteria that have been previously described. ${ }^{5}$ Whole sections were used for experiments in all specimens except 23 high-grade serous carcinomas that were arranged in tissue microarrays composed of $1 \mathrm{~mm}$ tissue cores in triplicate. The tissue collection was approved by the Institutional Review Board of the Johns Hopkins Medical Institutions and the Legacy Health System.

\section{Fluorescence In Situ Hybridization}

Two-color FISH assay was used to measure the gene copy number of CCNE1 per cell in serous tubal intraepithelial carcinoma and high-grade serous carcinoma as detailed previously. ${ }^{33}$ Briefly, 4- $\mu$ mthick sections were deparaffinized in xylene, hydrated through graded ethanol and incubated with proteinase $\mathrm{K}$ at $37^{\circ} \mathrm{C}$ for $33 \mathrm{~min}$. After washing in $2 \times$ Aniara saline-sodium citrate, the slides were placed in a denaturation solution $(70 \%$ formamide $/ 2 \times$ saline-sodium citrate) at $75^{\circ} \mathrm{C}$ for $5 \mathrm{~min}$, rinsed in 
$2 \times$ SSC. The slides were then dehydrated through graded ethanols, and dried in an oven at $62^{\circ} \mathrm{C}$ for 2 min. CCNE1/CEN19p FISH probe (cat\# FG0013, Abnova Corp, Taipei, Taiwan) was applied to the slides and coverslipped. DNA was denatured through incubation for $15 \mathrm{~min}$ at $80^{\circ} \mathrm{C}$, and hybridization was performed at $37^{\circ} \mathrm{C}$ for $20-24 \mathrm{~h}$. After washing for $20 \mathrm{~min}$ in $1.5 \mathrm{~mol} / \mathrm{l}$ urea in $0.2 \times \mathrm{SSC}$, slides were drained, dehydrated through graded ethanol, air-dried, mounted with ProLong ${ }^{\circledR}$ Gold Antifade Reagent with DAPI (cat\# P-36931, Invitrogen, Eugene, OR, USA) and imaged. The same assay was also applied to cell lines in culture including FT282 fallopian tube epithelial cells, OVCAR3, COV318, OVCA8, and OVSAHO ovarian cancer cells. OVCAR3 and COV318 were CCNE1-amplified lines while OVCAR8 has a CCNE1 gain and OVSAHO has a normal CCNE1 status. Percentage of cells with more than two centrosomes was estimated by counting at least six fields of view, each containing 20-100 randomly selected cells.

\section{Immunofluorescence}

Two-color immunofluorescence assay was used to measure the centrosome number per interphase cell in serous tubal intraepithelial carcinoma and highgrade serous carcinoma as previously described. ${ }^{34}$ Briefly, $5 \mu \mathrm{m}$ sections from each tissue block were deparaffinized in xylene then rehydrated through graded alcohols. Antigen retrieval was performed in Trilogy solution for $10 \mathrm{~min}$. The slides were then incubated at room temperature for $2 \mathrm{~h}$ with primary mouse-monoclonal $\gamma$-tubulin (T-5326, Sigma-Aldrich, dilution 1:200) and rabbit-monoclonal $\alpha$-tubulin (ab52866, Abcam, 1:200). Signal detection was performed by incubating the slides with fluorochromeconjugated secondary antibodies (from Jackson ImmunoResearch Laboratories) goat anti-mouse Rhodamine-Rd antibody (1:200), and goat anti-rabbit FITC (1:400) at room temperature for $1 \mathrm{~h}$. Three TBST washes were carried out before tissue sections were counterstained with DAPI and mounted.

\section{Image Analysis}

Three images from each serous tubal intraepithelial carcinoma, high-grade serous carcinoma, and normal fallopian tube were captured using a Nikon 50i epifluorescence microscope equipped with fluorescence excitation/emission filters for different fluorophores (Omega Optical) for both CCNE1 FISH and two-color immunofluorescence. Grayscale images were captured using Nikon NIS-Elements software and an attached Photometrics Cool snap EZ digital camera. For presentation purposes, images were pseudo-colored and merged. For each marker, three images from lesions were captured. Grayscale images were captured using Nikon NIS-Elements software and an attached Photometrics Cool snap EZ digital camera. The copy number was classified into five FISH strata. CCNE1 gain/amplification was defined as the presence of loose or tight CCNE1 signal cluster or $C C N E 1$ to centromeric probe (CEP19) ratio $\geq 2$ in more than $20 \%$ of the analyzed cells. CCNE1 copy number analysis was performed by counting FISH dot signals in 100 discrete nuclei for each lesion.

Centrosomes were visualized with immunofluorescent staining using the $\gamma$-tubulin antibody (labeled red), and mitotic spindles using the $\alpha$-tubulin antibody (labeled green). Centrosome amplification was defined as if there were more than 2 centrosomes per cell or if they were organized in large patchy aggregates (diameter $\geq 2 \mu \mathrm{m}$ ). For centrosome analysis, we performed counting in all discrete nuclei present in the pictures (minimum of 100) for each lesion to determine the fraction of tumor cells showing centrosome amplification.

\section{Statistical Analysis}

Statistical analysis was carried out using the GraphPad Prism software version 5.0 (GraphPad Software, San Diego, CA, USA). Comparisons of FISH findings between matched serous tubal intraepithelial carcinomas and high-grade serous carcinomas were obtained using the two-tailed Wilcoxon signed-rank test. The difference in the percentage of CCNE1 FISH- and centrosome-positive cases between serous tubal intraepithelial carcinomas and high-grade serous carcinomas was analyzed by paired twotailed $t$-test. Comparisons of CCNE1 copy number outcome with centrosomes were performed using the two-tailed Fisher's exact test. $P$-values of 0.05 or less were considered as statistically significant.

\section{Results}

CCNE1 copy number was classified into five categories-gain/amplification, high polysomy, low polysomy, trisomy, and disomy. In this study, CCNE1 gain/amplification and high polysomy were considered as FISH positive. We found that 8 (22\%) of 37 serous tubal intraepithelial carcinomas were CCNE1 FISH positive, of which 6 had amplification and 2 had high polysomy (Figure 1). Interestingly, 1 out of 7 serous tubal intraepithelial carcinomas that were not associated with high-grade serous carcinoma showed CCNE1 high polysomy (FISH positive). For high-grade serous carcinomas, 12 (28\%) of 43 cases were CCNE1 FISH positive, including 10 with amplification and 2 with high polysomy (Figure 1). In this series, 30 serous tubal intraepithelial carcinomas were associated with 19 high-grade serous carcinomas, among which 11 have a second serous tubal intraepithelial carcinoma (Table 1). We found a significant concordance in CCNE1 copy number (FISH-positive vs FISH-negative cases) between serous tubal intraepithelial carcinoma and high-grade serous carcinoma from the same 19 

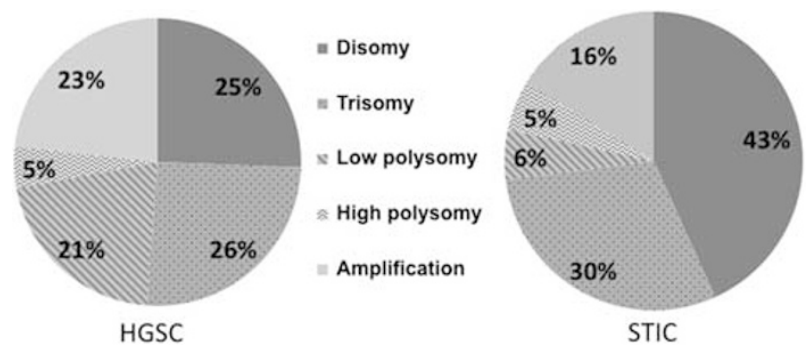

Figure 1 Summary of CCNE1 FISH results in ovarian high-grade serous carcinoma and serous tubal intraepithelial carcinoma. The results are classified into five categories, and their percentages in different lesions are shown.

Table 1 FISH analysis of CCNE1 copy number in 19 primary HGSCs and concurrent STICs

\begin{tabular}{lccc}
\hline $\begin{array}{l}\text { Copy number } \\
\text { category }\end{array}$ & $\begin{array}{c}\text { HGSC, } n \\
(\%)\end{array}$ & $\begin{array}{c}\text { First STIC, } n \\
(\%)\end{array}$ & $\begin{array}{c}\text { Second STIC, } \\
n(\%)\end{array}$ \\
\hline FISH negative & $14(74)$ & $14(74)$ & $9(81)$ \\
$\quad$ Disomy & $5(26)$ & $6(32)$ & $4(36)$ \\
$\quad$ Trisomy & $5(26)$ & $7(37)$ & $4(36)$ \\
Low polysomy & $4(21)$ & $1(5)$ & $1(9)$ \\
FISH positive & $5(26)$ & $5(26)$ & $2(19)$ \\
$\quad$ High polysomy & $1(5)$ & $1(5)$ & $2(19)$ \\
$\quad$ Amplification & $4(21)$ & $4(21)$ & $11(100)$ \\
Total & $19(100)$ & $19(100)$ & \\
\hline
\end{tabular}

patients $(P<0.001)$. In high-grade serous carcinomas showing FISH positive $(n=5)$, their associated serous tubal intraepithelial carcinomas were also FISH positive while in 14 FISH-negative high-grade serous carcinomas, none of their associated serous tubal intraepithelial carcinomas were FISH positive (Table 1). There was no significant difference in the percentage of CCNE1 FISH-positive serous tubal intraepithelial carcinomas compared with CCNE1 FISH-positive high-grade serous carcinomas $(P=0.613$, Chi square). No evidence of CCNE1 copy number changes was noted in normal-appearing fallopian tube epithelium adjacent to or remote from serous tubal intraepithelial carcinomas. A representative serous tubal intraepithelial carcinoma showing CCNE1 amplification is shown in Figure 2. There were 20 high-grade serous carcinomas examined on whole sections and 23 high-grade serous carcinomas in tissue microarrays and there was a strong correlation in percentage of CCNE1 copy number changes between whole section and tissue microarrays $\left(r^{2}=0.993, P<0.05\right)$, indicating that tissue microarray format was compatible with FISH analysis.

To measure the centrosome number, we applied a double-color immunofluorescence for $\gamma$-tubulin and $\alpha$-tubulin to simultaneously visualize centrosomes and microtubules, respectively. Only whole sections were stained for centrosome immunofluorescence. Centrosome amplification (centrosome number $>2$ ) was significantly increased in high-grade serous carcinoma as compared with serous tubal intraepithelial carcinoma $(P=0.0006$, Wilcoxon rank test $)$ as it was recorded in only $5(14 \%)$ of 37 serous tubal

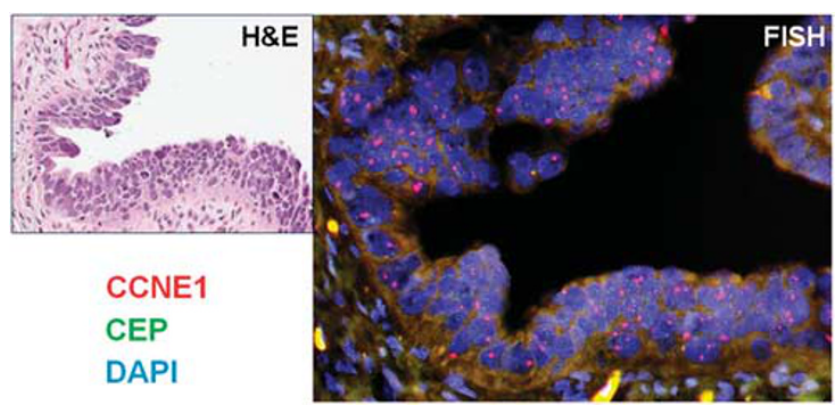

Figure 2 CCNE1 two-color FISH in a representative serous tubal intraepithelial carcinoma. The H\&E of the lesion is shown at the left panel. Red signals: CCNE1 probe; green signal: CEP control probe; blue: nuclear staining with DAPI. There are many serous tubal intraepithelial carcinoma cells exhibiting CCNE1 amplification (big red dots).

intraepithelial carcinomas, in contrast to 10 (40\%) of 25 high-grade serous carcinomas (Figure 3a and b). A representative serous tubal intraepithelial carcinoma showing centrosome amplification is illustrated in Figure 4. A pair-wise comparison of normal fallopian tube epithelium, serous tubal intraepithelial carcinoma, and high-grade serous carcinoma from the same cases demonstrated that 21 (88\%) of 24 highgrade serous carcinomas had a higher percentage of cells showing centrosome amplification than corresponding serous tubal intraepithelial carcinomas and three of the high-grade serous carcinoma demonstrated a mild decrease in percentage of cells with centrosome amplification as compared with the matched serous tubal intraepithelial carcinomas (Figure 3c). In general, there was a positive correlation in percentage of cells with centrosome amplification between high-grade serous carcinoma and serous tubal intraepithelial carcinoma from the same patients (Figure 3d). We did not observe any abnormal centrosome number in normal-appearing fallopian tube epithelial or stromal cells.

To determine whether cyclin E1 upregulation resulted in centrosome amplification, we used an epithelial cell line, FT282, established from a normal fallopian tube as previously described. ${ }^{27}$ Derivative cell lines (FT282-V, FT282-CE) were generated using pMSCV-neo-(empty) and pMSCV-neo CCNE1, encoding full-length CCNE1 subcloned from pRc/ CMV 7946. We found that as compared with FT282$\mathrm{V}$ control cells, FT282-CE cells expressing more cyclin E1 had significantly higher percentage of cells with more than two centrosomes (ie, centrosome amplification) (Figure 5). The percentage of amplified cells in FT282-CE was similar to ovarian cancer cell lines (Figure 5).

\section{Discussion}

Elucidating the molecular alterations at an early stage of tumor development has greatly enhanced our understanding of cancer pathogenesis which 

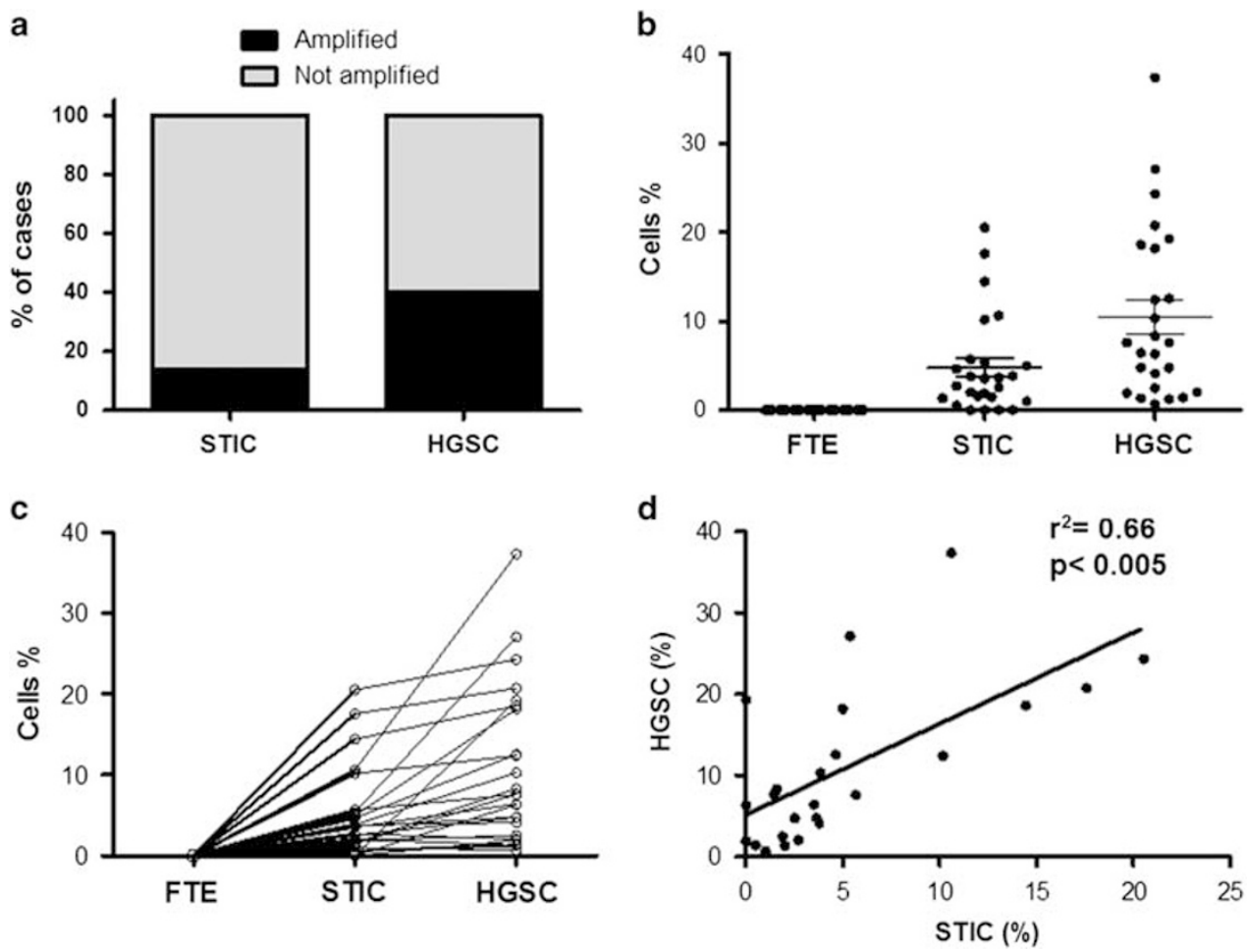

Figure 3 Centrosome numbers were determined by immunofluorescence in high-grade serous carcinoma (HGSC), serous tubal intraepithelial carcinoma (STIC) and normal fallopian tube epithelium (FTE). (a) Percentage of lesions showing centrosome amplification in 33 STICs and 25 HGSCs. (b) Percentage of tumor cells demonstrating centrosome amplification. All specimens containing normal fallopian tube epithelium do not have centrosome amplification. (c) Percentage of tumor cells with centrosome amplification in paired STIC and the corresponding HGSC. (d) A positive correlation of percentage of tumor cells showing centrosome amplification between STICs and HGSCs $\left(r^{2}=0.65, P<0.005\right)$.

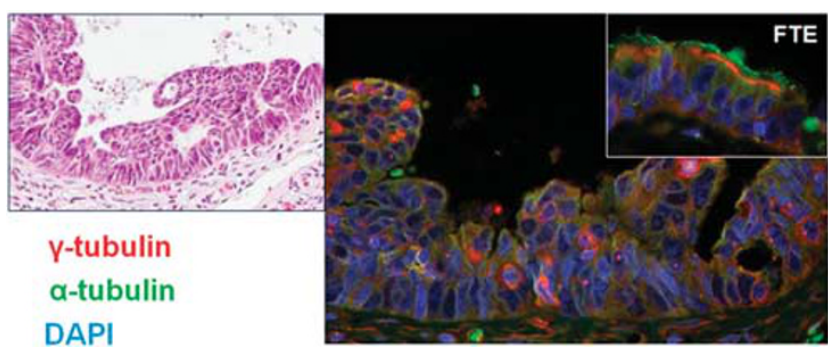

Figure 4 Detection of centrosomes using immunofluorescence in a representative serous tubal intraepithelial carcinoma. The H\&E of the lesion is shown at the left panel. Red signals: $\gamma$-tubulin (stain centrosomes); green signal: $\alpha$-tubulin (stains cilia); blue: nuclear staining with DAPI. There are several cells containing big red dots (ie, centrosome amplification) in serous tubal intraepithelial carcinoma cells at the interface but not in normal fallopian tube epithelium (FTE, inset).

will, in turn, have an important implication on early detection, diagnosis, and prevention. The molecular analysis of serous tubal intraepithelial carcinomas has been difficult and challenging as it depends on genome-wide assays, which usually require a substantial amount of lesion cells. The diagnosis of these extremely minute lesions is made after histological examination following formalin fixation and paraffin embedding; and therefore, fresh tissue is not available for conventional gene expression analysis. To circumvent these limitations, we employed FISH and immunofluorescence to compare CCNE1 copy number and centrosome number between serous tubal intraepithelial carcinoma and high-grade serous carcinoma. The results of this study provide new evidence that $C C N E 1$ copy number gain/amplification occurs early in ovarian tumorigenesis, ie, in serous tubal intraepithelial carcinoma, while centrosome amplification appears to represent a later molecular event. This finding supports the view that serous tubal intraepithelial carcinoma is likely a precursor of many ovarian high-grade serous carcinomas as serous tubal intraepithelial carcinomas shows less frequent centrosome amplification than high-grade serous carcinoma.

The finding of a similar frequency of CCNE1 gain/ amplification in both serous tubal intraepithelial carcinoma and high-grade serous carcinoma together with our previous reports ${ }^{26,27}$ showing cyclin E1 overexpression in serous tubal intraepithelial carcinomas but not in adjacent fallopian tube epithelium strongly suggests that cyclin E1 upregulation occurs in the precursor stage of many high-grade serous carcinomas. This observation is similar to those in our report showing a comparable frequency of CCNE1 gain/amplification (41-45\%) in uterine serous carcinoma and its precursor serous endometrial intraepithelial carcinoma. ${ }^{33}$ It is known that there is 


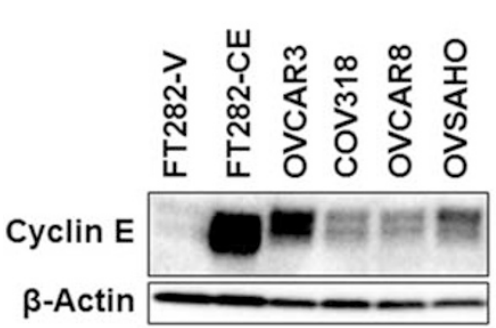

b

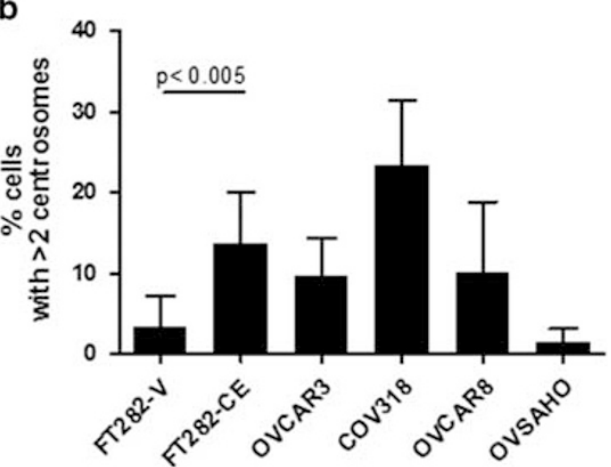

Figure 5 The effect of cyclin E1 expression on centrosome numbers on fallopian tube epithelial cells. (a) Western blot analysis shows protein levels of cyclin E1 in fallopian tube epithelial cells (FT282-V) and cyclin E1 expressing cells (FT282-CE), which were transfected by a plasmid expressing CCNE1 gene. Cyclin E1 expression in other ovarian cancer cell lines is also shown. (b) The percentage of cancer cells with centrosome amplification ( $>2$ centrosomes/tumor nucleus) in each cell line. FT282-CE cells have significantly higher percentage of cells with centrosome amplification than its parental FT282-V cells $(P<0.005)$.

a positive correlation between CCNE1 copy number and mRNA levels in ovarian high-grade serous carcinoma (The Cancer Genome Atlas ovarian cancer data set). Therefore, it was expected that those serous tubal intraepithelial carcinomas with CCNE1 gain/ amplification would express more cyclin E1. Cyclin E1 alone may not be able to transform nontumorigenic epithelial cells ${ }^{23}$ but may act in concert with other molecules to promote tumor development as induced co-expression of cyclin E1 and Rsf-1, another ovarian cancer-associated gene. ${ }^{35}$ Of note, Rsf-1 is also frequently amplified and overexpressed in high-grade serous carcinoma and serous tubal intraepithelial carcinoma, and both cyclin E1 and Rsf-1 interact with each other to promote tumor growth. ${ }^{35}$ Thus, it is likely that cyclin E1 contributes to the genesis of serous tubal intraepithelial carcinoma by transforming normal tubal epithelial cells through collaborating with Rsf-1, mutant p53 and perhaps other molecular alterations.

The role of cyclin E1 in promoting chromosomal instability has been established in cancer cells, ${ }^{21,36,37}$ and the result from this study demonstrating that induced expression of cyclin E1 increased the percentage of non-transformed fallopian tube epithelial cells showing centrosome amplification further supports this view. However, it should not be construed that cyclin E1 upregulation is the only mechanism contributing to chromosomal instability. For example, defective homologous recombination pathway for DNA double-strand break repair and telomere attrition can also cause chromosomal instability. To that end, it has been reported that CCNE1 gain/amplification and mutations in homologous recombination repair genes such as $B R C A 1 / 2$ are, in general, mutually exclusive, suggesting that the high level of chromosomal instability in highgrade serous carcinoma results either from aberration of the cyclin E1 pathway or the BRCA1/2 pathway. ${ }^{23}$

Another interesting observation in this study was the demonstration of greater centrosome numbers per tumor cell in high-grade serous carcinoma than in serous tubal intraepithelial carcinoma. Centrosome amplification is considered as a surrogate marker of chromosomal instability because more than two centrosomes readily induce unbalanced chromosomal segregation after cell division. Accordingly, the higher level of chromosomal instability in high-grade serous carcinoma compared with serous tubal intraepithelial carcinoma suggests that serous tubal intraepithelial carcinoma precedes high-grade serous carcinoma. It has been suggested that serous tubal intraepithelial carcinomas may represent lateral extension from the invasive high-grade serous carcinoma or that serous tubal intraepithelial carcinomas are metastases but either of these processes would demonstrate similar centrosome numbers in both the high-grade serous carcinoma and the serous tubal intraepithelial carcinoma. Accordingly, our findings provide evidence that serous tubal intraepithelial carcinoma is likely the immediate precursor of highgrade serous carcinoma. In a previous study using telomere FISH, we were able to demonstrate short telomeres in serous tubal intraepithelial carcinoma compared with high-grade serous carcinoma; short telomeres have been demonstrated to be one of the earliest molecular changes in carcinogenesis. ${ }^{17}$ Thus, shorter telomere and lower frequency of centrosome amplification in serous tubal intraepithelial carcinoma compared with high-grade serous carcinoma together with the finding that ALDH1A1 is expressed in high-grade serous carcinoma but not in serous tubal intraepithelial carcinoma ${ }^{38}$ support the proposal that serous tubal intraepithelial carcinomas are precursors of many high-grade serous carcinomas.

There are several limitations in the current study that should be acknowledged. First, a number of high-grade serous carcinomas failed to show an increased fraction of cells harboring centrosome amplification as compared with their corresponding serous tubal intraepithelial carcinomas; and therefore, they were considered uninformative. In these cases, this may have been the result of sampling bias due to a relatively small number of serous tubal 
intraepithelial carcinoma cells available for analysis. Alternatively, it is conceivable that not all serous tubal intraepithelial carcinomas are the precursors of the high-grade serous carcinomas so the centrosome numbers are similar between metastasis morphologically resembling serous tubal intraepithelial carcinoma and high-grade serous carcinoma. Second, geographical variation in centrosome numbers can be present because of intra-tumoral heterogeneity. The high-grade serous carcinoma areas we analyzed may represent a focal increase in centrosome number as compared with serous tubal intraepithelial carcinoma. Considering this potential problem, we attempted to sample high-grade serous carcinoma areas that were in the fallopian tube or close to the serous tubal intraepithelial carcinoma. Finally, we did not find any correlation between CCNE1 gain/ amplification and centrosome amplification in our cases although CCNE1 amplification is thought to cause centrosome amplification. Similarly, we did not observe an association between the percentage of ovarian cancer cells showing centrosome amplification and CCNE1 amplification and overexpression in cell lines (Figure 5). This result suggests that in the context of high-grade serous carcinoma pathogenesis, redundant mechanisms other than cyclin E1 pathway exist for numeric regulation of centrosomes in tumor cells such as de novo synthesis of centrosomes. ${ }^{39}$

In summary, the findings of this study demonstrate that CCNE1 copy number gain/amplification occurs in $22 \%$ of serous tubal intraepithelial carcinomas that are associated with high-grade serous carcinomas, suggesting that amplification of CCNE1 serves as one mechanism for the development of some serous tubal intraepithelial carcinomas. Moreover, centrosome amplification in tumor cells is more frequently detected in high-grade serous carcinomas than in serous tubal intraepithelial carcinomas, indicating a progressive acquisition of chromosomal instability during tumor progression lending further support to the hypothesis that many high-grade serous carcinomas arise from serous tubal intraepithelial carcinomas.

\section{Acknowledgments}

This study is supported in part by a grant from US Department of Defense W81XWH-11-2-0230/ OC100517 (to RJK, IMS, and TLW), the National Institute of Health CA165807 (to TLW), the Ephraim \& Wilma Shaw Roseman Foundation (to IMS) and the HERA Women Cancer Foundation (to EK); The $\mathrm{Dr}$ Miriam and Sheldon G. Adelson Medical Research Foundation (to RD), a DOD OCRP award (OC140511 to RD), and a grant from the German DFG Research Foundation (to KD).

\section{Disclosure/conflict of interest}

The authors declare no conflict of interest.

\section{References}

1 Siegel RL, Miller KD, Jemal A. Cancer statistics, 2015. CA Cancer J Clin 2015;65:5-29.

2 Kurman RJ, Shih IM. The dualistic model of ovarian carcinogenesis. revisited, revised and expanded. Am J Pathol 2016;186:733-747.

3 Crum CP, Drapkin R, Miron et al. The distal fallopian tube: a new model for pelvic serous carcinogenesis. Curr Opin Obstet Gynecol 2007;19:3-9.

4 Perets R, Wyant GA, Muto KW et al. Transformation of the fallopian tube secretory epithelium leads to highgrade serous ovarian cancer in Brca;Tp53;Pten models. Cancer Cell 2013;24:751-765.

5 Visvanathan K, Vang R, Shaw $\mathrm{P}$ et al. Diagnosis of serous tubal intraepithelial carcinoma based on morphologic and immunohistochemical features: a reproducibility study. Am J Surg Pathol 2011;35: 1766-1775.

6 Kuhn E, Kurman RJ, Soslow RA et al. The diagnostic and biological implications of laminin expression in serous tubal intraepithelial carcinoma. Am J Surg Pathol 2012;36:1826-1834.

7 Karst AM, Levanon K, Duraisamy S et al. Stathmin 1, a marker of PI3K pathway activation and regulator of microtubule dynamics, is expressed in early pelvic serous carcinomas. Gynecol Oncol 2011;123:5-12.

8 Novak M, Lester J, Karst AM et al. Stathmin 1 and p16 (INK4A) are sensitive adjunct biomarkers for serous tubal intraepithelial carcinoma. Gynecol Oncol 2015; 139:104-111.

9 Kindelberger DW, Lee Y, Miron et al. Intraepithelial carcinoma of the fimbria and pelvic serous carcinoma: Evidence for a causal relationship. Am J Surg Pathol 2007;31:161-169.

10 Przybycin CG, Kurman RJ, Ronnett BM et al. Are all pelvic (nonuterine) serous carcinomas of tubal origin? Am J Surg Pathol 2010;34:1407-1416.

11 Morrison JC, Blanco LZ Jr., Vang R et al. Incidental serous tubal intraepithelial carcinoma and early invasive serous carcinoma in the nonprophylactic setting: analysis of a case series. Am J Surg Pathol 2015;39: 442-453.

12 Gilks CB, Irving J, Kobel $\mathrm{M}$ et al. Incidental nonuterine high-grade serous carcinomas arise in the fallopian tube in most cases: further evidence for the tubal origin of high-grade serous carcinomas. Am J Surg Pathol 2015;39:357-364.

13 Hirst JE, Gard GB, McIllroy K et al. High rates of occult fallopian tube cancer diagnosed at prophylactic bilateral salpingo-oophorectomy. Int J Gynecol Cancer 2009;19:826-829.

14 Rabban JT, Garg K, Crawford B et al. Early detection of high-grade tubal serous carcinoma in women at low risk for hereditary breast and ovarian cancer syndrome by systematic examination of fallopian tubes incidentally removed during benign surgery. Am J Surg Pathol 2014;38:729-742.

15 Tang S, Onuma K, Deb P et al. Frequency of serous tubal intraepithelial carcinoma in various gynecologic malignancies: a study of 300 consecutive cases. Int J Gynecol Pathol2012;31:103-110.

16 Kuhn E, Kurman RJ, Vang R et al. TP53 mutations in serous tubal intraepithelial carcinoma and concurrent pelvic high-grade serous carcinoma- evidence supporting the clonal relationship of the two lesions. J Pathol 2012;226:421-426. 
17 Kuhn E, Meeker A, Wang TL et al. Shortened telomeres in serous tubal intraepithelial carcinoma: an early event in ovarian high-grade serous carcinogenesis. Am J Surg Pathol 2010;34:829-836.

18 Sherman-Baust CA, Kuhn E, Valle BL et al. A genetically engineered ovarian cancer mouse model based on fallopian tube transformation mimics human high-grade serous carcinoma development. J Pathol 2014;233:228-237.

19 Kobayashi Y, Kashima H, Wu RC et al. Mevalonate pathway antagonist inhibits proliferation of serous tubal intraepithelial carcinoma and ovarian carcinoma in mouse models. Clin Cancer Res 2015;21:4625-4662.

20 McDaniel AS, Stall JN, Hovelson DH et al. Nextgeneration sequencing of tubal intraepithelial carcinomas. JAMA Oncol 2015;1:1128-1132.

21 Fukasawa K. Centrosome amplification, chromosome instability and cancer development. Cancer Lett 2005;230:6-19.

22 Cancer Genome Atlas Research Network. Integrated genomic analyses of ovarian carcinoma. Nature 2011; 474:609-615.

23 Patch AM, Christie EL, Etemadmoghadam D et al. Whole-genome characterization of chemoresistant ovarian cancer. Nature 2015;521:489-494.

24 Etemadmoghadam D, George J, Cowin PA et al. Amplicon-dependent CCNE1 expression is critical for clonogenic survival after cisplatin treatment and is correlated with 20q11 gain in ovarian cancer. PLoS One 2010;5:e15498.

25 Nakayama K, Nakayama N, Jinawath N et al. Amplicon profiles in ovarian serous carcinomas. Int J Cancer 2007;120:2613-2617.

26 Sehdev AS, Kurman RJ, Kuhn E et al. Serous tubal intraepithelial carcinoma upregulates markers associated with high-grade serous carcinomas including Rsf-1 (HBXAP), cyclin E and fatty acid synthase. Mod Pathol 2010;23:844-855.

27 Karst AM, Jones PM, Vena N et al. Cyclin E1 deregulation occurs early in secretory cell transformation to promote formation of fallopian tube-derived high-grade serous ovarian cancers. Cancer Res 2014;74:1141-1152.

28 Farley J, Smith LM, Darcy KM et al. Cyclin E expression is a significant predictor of survival in advanced, suboptimally debulked ovarian epithelial cancers: a Gynecologic Oncology Group study. Cancer Res 2003;63:1235-1241.

29 Rosen DG, Yang G, Deavers MT et al. Cyclin E expression is correlated with tumor progression and predicts a poor prognosis in patients with ovarian carcinoma. Cancer 2006;106:1925-1932.

30 Davidson B, Skrede M, Silins I et al. Low-molecular weight forms of cyclin $\mathrm{E}$ differentiate ovarian carcinoma from cells of mesothelial origin and are associated with poor survival in ovarian carcinoma. Cancer 2007;110:1264-1271.

31 Nigg EA, Stearns T. The centrosome cycle: centriole biogenesis, duplication and inherent asymmetries. Nat Cell Biol 2011;13:1154-1160.

32 Cope L, Wu RC, Shih Ie M et al. High level of chromosomal aberration in ovarian cancer genome correlates with poor clinical outcome. Gynecol Oncol 2013;128:500-505.

33 Kuhn E, Bahadirli-Talbott A, Shih Ie M. Frequent CCNE1 amplification in endometrial intraepithelial carcinoma and uterine serous carcinoma. Mod Pathol 2014;27:1014-1019.

34 Kuhn E, Ayhan A, Shih Ie M et al. Ovarian Brenner tumour: a morphologic and immunohistochemical analysis suggesting an origin from fallopian tube epithelium. Eur J Cancer 2013;49:3839-3849.

35 Sheu JJ, Choi JH, Guan B et al. Rsf-1, a chromatin remodelling protein, interacts with cyclin E1 and promotes tumour development. J Pathol 2013;229: 559-568.

36 Spruck CH, Won KA, Reed SI. Deregulated cyclin E induces chromosome instability. Nature 1999;401: 297-300.

37 Bagheri-Yarmand R, Biernacka A, Hunt KK et al. Low molecular weight cyclin E overexpression shortens mitosis, leading to chromosome missegregation and centrosome amplification. Cancer Res 2010;70: 5074-5084.

38 Chui $\mathrm{MH}$, Wang Y, Wu RC et al. Loss of ALDH1A1 expression is an early event in the pathogenesis of ovarian high-grade serous carcinoma. Mod Pathol 2015;28:437-445.

39 Loncarek J, Khodjakov A. Ab ovo or de novo? Mechanisms of centriole duplication. Mol Cells 2009; 27:135-142. 